\title{
Endoprothetik bei Erwachsenen mit juveniler idiopathischer Arthritis
}

M. Arbogast' ${ }^{1}$ J.-P. Haas ${ }^{2}$

${ }^{1}$ Abteilung für Rheumaorthopädie und Handchirurgie, Zentrum für Rheumatologie, Orthopädie und Schmerztherapie, Klinik Oberammergau, Oberammergau; ${ }^{2}$ Deutsches Zentrum für Kinder- und Jugendrheumatologie, Garmisch-Partenkirchen

\section{Schlüsselwörter}

Endoprothetik junger Rheumatiker, operative Therapie bei progressiver JIA

\section{Zusammenfassung}

Progressive Verlaufsformen der JIA, die durch medikamentöse Behandlung wenig beeinflussbar sind, münden nicht selten in eine endoprothetische Versorgung vor dem fünften Lebensjahrzent. Durch den Beginn im Kindesoder Jugendalter werden Wachstum und physiologische Gelenkentwicklung verändert. Daraus resultierende Bewegungseinschränkungen und Gelenkfehlstellungen beeinflussen Implantatwahl und Weichteilbehandlung. Schmerzlinderung und Verbesserung der Lebensqualität nach endoprothetischer Versorgung sind beeindruckend. Dennoch ist die Lebensplanung mit Endoprothesen unterschiedlich zu prognostizieren und abhängig vom Implantationsort.

Korrespondenzadresse

Dr. Martin Arbogast

Abteilung für Rheumaorthopädie und Handchirurgie

Zentrum für Rheumatologie, Orthopädie und Schmerz-

therapie

Klinik Oberammergau

Hubertusstraße 40, 82487 Oberammergau

Tel.: 08822/914-311, Fax: 08822-914-162

E-Mail: martin.arbogast@Wz-kliniken.de

\section{Keywords}

Endoprosthetics of young rheumatism, operative therapy in progressive JIA

\section{Summary}

Progressive forms of JIA, which are not good enough influenced by drug treatment, can lead to an endoprosthetic treatment bevor the $5^{\text {th }}$ lifedecade. Beginning in childhood or adolescence, growth and physiological joint developement are changed. The resulting movement restrictions and joint deformities influence implant selection and soft tissue treatment. Pain relief and improvement in quality of live after endoprosthetics care are impressive. Nevertheless, life planning with endoprostheses are different from prognoses and depend on the joint region.

Endoprosthetic treatment in adults with juvenile idiopathic arthritis

arthritis + rheuma 2018; 38: 273-281 oligoartikuläre JIA [EO-JIA], seronegative polyartikuläre JIA, seropositve polyartikuläre JIA, Enthesitis-assoziierte JIA [EAJIA], Psoriasis-Arthritis [PsJIA] und undifferenzierte JIA) unterschieden. Bei der oligoartikulären JIA kann die definitive $\mathrm{Zu}$ ordnung erst nach mindestens sechs Monaten Erkrankungsverlauf erfolgen, weil erst im Verlauf eine Einteilung in die Unterformen persistierende (maximal vier betroffene Gelenke) und extended (mehr als vier Gelenke betroffen) erfolgen kann. Auch beim Vorliegen einer zunächst undifferenzierten JIA kann die endgültige Klassifikation oft erst im Verlauf möglich sein (20). Die JIA hat in den meisten Fällen einen Verlauf über Jahre, der entweder durch eine chronische Aktivität oder einen schubweisen Verlauf gekennzeichnet ist. Die Folgen der Arthritis für die betroffenen Gelenke und die umliegenden Strukturen können unmittelbar, jedoch auch langfristig, d.h. als Folge von Gelenksfunktionsstörungen und -abnutzungen auftreten. Extraartikuläre Manifestationen (EAM) sind möglich und erfordern z. T. ein besonderes Vorgehen. Die Erkrankung kann die Entwicklung der betroffenen Kinder und Jugendlichen sowohl bezüglich des Wachstums wie auch bezüglich neuromotorischer Fähigkeiten erheblich beeinträchtigen. Die Therapie einer JIA kann sich daher nicht ausschließlich am Subtypen orientieren, sondern muss den Grad der Aktivität, EAM, den individuellen Verlauf, funktionelle Defizite, Alter und Entwicklungsstand des Patienten miteinbeziehen. Dies erfordert die Versorgung der Patienten durch speziell geschulte Kinder- und Jugendrheumatologen. Der Übergang ins Erwachsenenalter gestaltet sich für junge Rheumatiker nicht immer elegant, weswegen die kontinuierliche Behandlung noch aktiver Gelenkregionen nicht immer adäquat therapiert wird. $40-60 \%$ der entzündautoimmunologische und/oder autoinflammatorische Ursachen haben können. Insgesamt gehen wir von ca. 22-25000 betroffenen Kindern und Jugendlichen in der BRD aus. Während einige rheumatologische Erkrankungsbilder des Kindes- und Jugendalters extrem selten sind, findet sich der kindliche Gelenkrheumatismus - juvenile idiopathische Arthritis (JIA) - mit

über 15000 betroffenen Patienten relativ haufig. Als JIA wird eine Arthritis mit gefunden werden können. Entsprechend der klinischen Manifestation werden gemäß der Klassifikationskriterien der International League of Association for Rheumatology (27) acht verschiedene Unterformen (Systemische JIA [S-JIA], persistierende oligoartikuläre JIA [PO-JIA], extended 
lich rheumatischen Erkrankungen im Kindes- und Jugendalter persistieren bei Erwachsenen mit JIA (10). Dies führt nicht selten zu einem therapieresistenten Gelenk, welches dann endoprothetisch versorgt werden muss. Durch die verbesserte medikamentöse Therapie der JIA scheint die Zahl der implantierten Gelenke bei der JIA tendenziell abzunehmen und der Altersdurchschnitt steigt an (26).

\section{Indikation und Vorausset- zungen}

Grundsätzlich sind sekundär destruierte Gelenke bei der JIA im Durchschnitt früher zu ersetzen als bei primär degenerativen Erkrankungen (31). Dies trifft für alle Gelenkabschnitte von oberen und unteren

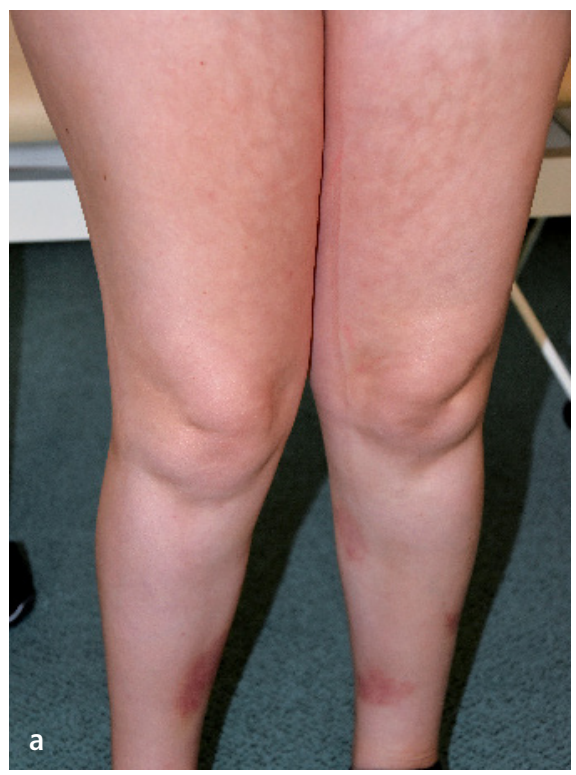

genannte Basismedikation wieder aufgenommen werden, um ein Rebound der Erkrankung zu vermeiden. Um eine Lebensplanung mit Endoprothesen verbunden mit Wechseloperationen zu realisieren, sind knochensparende Modelle vorzuziehen. Ob zementiert oder zementfrei hängt von der Knochenfestigkeit der Gelenkregion ab und sollte individuell entschieden werden. Signifikante Unterschiede zwischen zementiert und zementfrei bestehen weder in der Hüft- noch in Knieendoprothetik (38).

\section{Technik und Gelenkregion}

Der „golden standard“ in der Schultergelenkendoprothetik ist die Hemiarthroplastik. Zufriedenstellende Schmerzreduktion

Abb. 1 (a-d) klinisches Bild präoperativ und radiologische Veränderungen Larsen $\mathrm{V}$
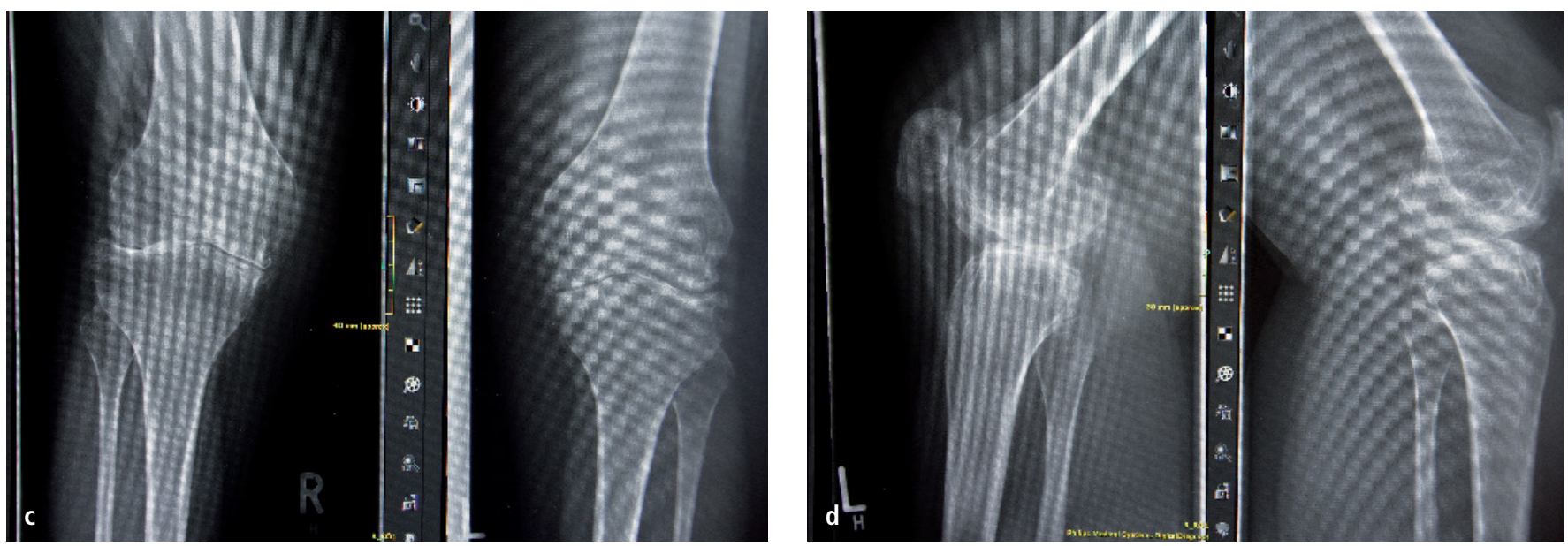
und moderate Bewegungsverbesserung $(13,14,36)$ sind gegenüber den erhöhten Ausbauraten der Pfanne wegen einer aseptischen Lockerung vorteilhaft (13). Ist jedoch die Rotatorenmanschette subtotal oder total defekt, kann eine inverse Endoprothetik Anwendung finden mit interessanten mittelfristigen Ergebnissen. Da bei den jungen Rheumatikern mit einer Hemiendoprothese eine zunehmende Defektbildung der Supraspinatussehne eintritt, ist die inverse Vorgehensweise auch als primäre Maßnahme durchaus zu überdenken. Die Standzeiten sind vielversprechend (14, 22).
Deutlich seltener ist die Indikation zu einer Ellenbogenendoprothese, die erhöhte Revisionsraten aufzeigt (3), dennoch auch erfreuliche Langzeitergebnisse liefern kann (3).

Auch hier sind Schmerzreduktion und Bewegungsverbesserung wegweisend $(2,3)$.

Am Handgelenk und an den Fingergrund und -mittelgelenken ist die Endoprothetik bei der JIA unterrepräsentiert und hat nur Einzelfallcharakter (38). Eine Tendenz zur Indikation und Verlaufsbeobachtung zu treffen, ist wegen der geringen Literaturangaben schwierig.

Anders bei der Hüft- und Knieendoprothetik: Hier existieren zahlreiche Studien, die insbesondere einen Anstieg der Le- bensqualität mit Schmerzreduktion und Verbesserung der Beweglichkeit und Belastungsfähigkeit der unteren Extremitäten beweisen $(4,5,7,15,16)$.

Auch sind die Standzeiten in der Knieendoprothetik mit 97 bis $99 \%$ in den ersten fünf bis sechs Jahren $(6,23)$ eindrucksvoll und zeigen den hohen Stellenwert der Endoprothetik. Langzeituntersuchungen von 20 Jahren sind jedoch mit einem Rückgang der Standzeiten auf 58,5\% verbunden (24). Die Revisionsprobleme an der Hüfte sind nicht unerheblich (9) und gehen mit einer höheren Komplikationsrate einher. Bei sparsamer Knochenresektion ist eine Lebensplanung mit der Endoprothetik mit
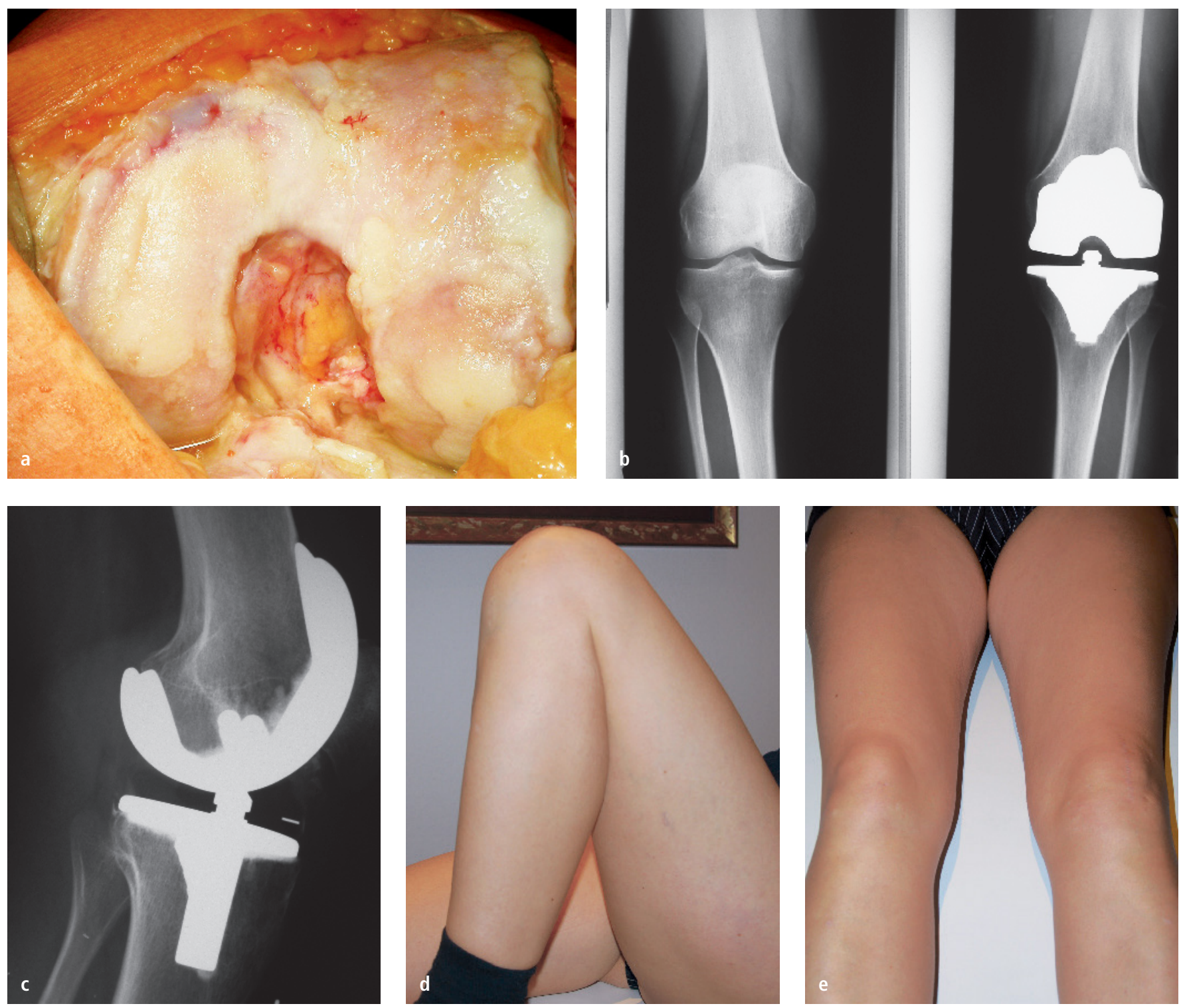

Abb. 2 (a-e) intraoperatives Bild, radiologische und klinische Kontrolle nach 13 Jahren 

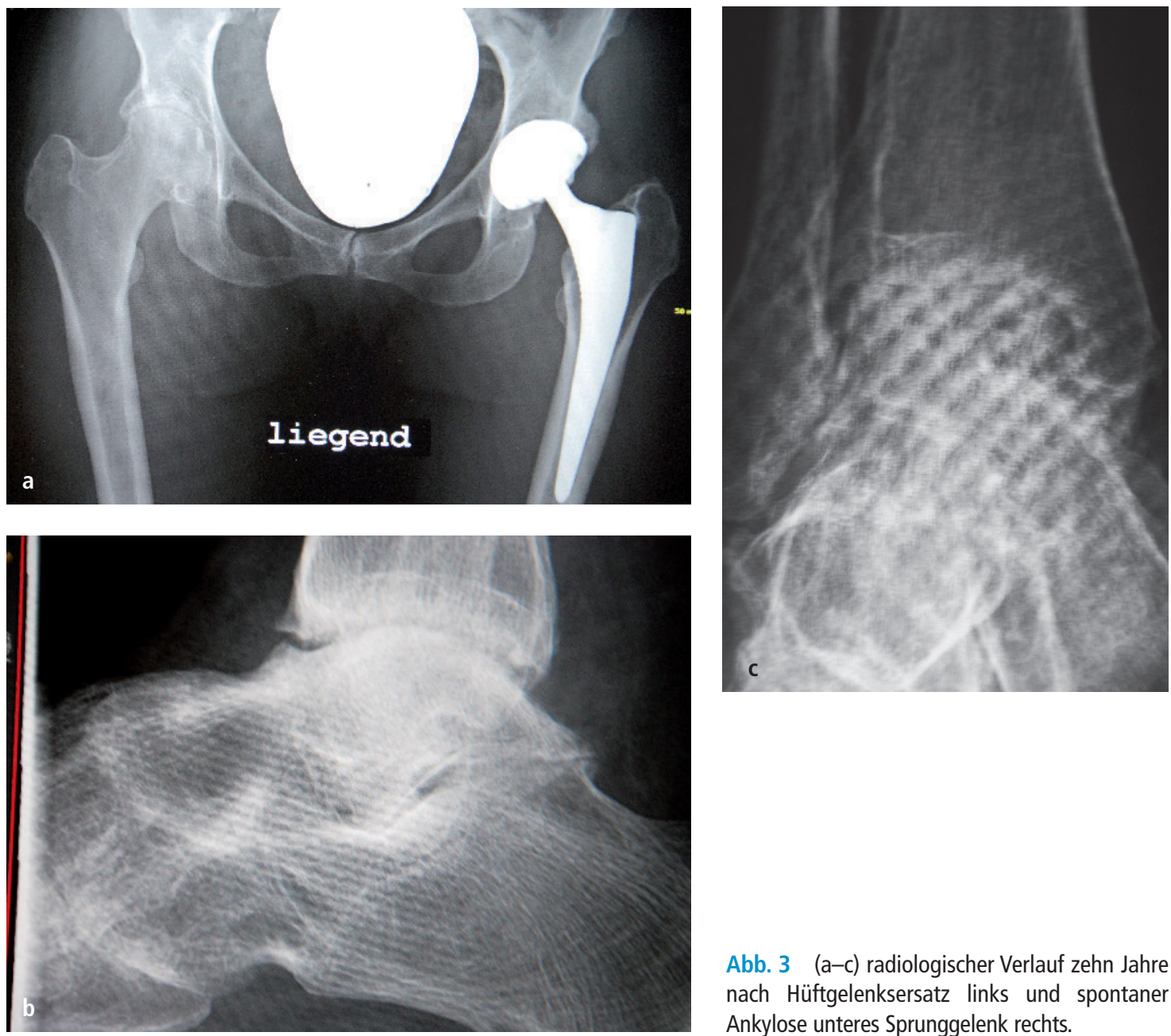

Abb. 3 (a-c) radiologischer Verlauf zehn Jahre nach Hüftgelenksersatz links und spontaner Ankylose unteres Sprunggelenk rechts.

ein oder zwei Wechseloperationen nicht unwahrscheinlich. Ein deutlicher Anstieg der Lebensqualität ist zu verzeichnen (15, 24, 28)

Vorfuß und Sprunggelenk spielen in der Endoprothetik bei der JIA eine untergeordnete Rolle, Studien mit signifikanten Fallzahlen gibt es nicht (34).

\section{Fallbeispiele}

\section{Fall 1}

22-jährige junge Frau mit seit dem dritten Lebensjahr bestehender JIA mit polyartikulärem Verlauf. Teileffektiv mit zuletzt Infliximab, Tocilizumab, Rituximab, Etanercept, MTX. Gehstrecke circa 100 Meter, nicht beschwerdefrei, VAS 8-10. Augenmitbeteiligung, eingeschränkter Bewegungsumfang des Ellenbogengelenkes rechts, rezidivierender schubartiger Ver- lauf. Kniedendoprothetik rechts geplant, nach Rehabilitation auch links ( $>$ Abb. 1).

\section{Fall 2}

39-jährige junge Frau mit JIA seit dem dritten Lebensjahr mit oliarthritischer Verlaufsform an beiden Knie- und Sprunggelenken. Progressiver Verlauf am linken Knie, arthroskopische Synovialektomie mit 18 Jahren, mit 26 Jahren bikondylärer Oberflächenersatz, seither keine Schmerzen im linken Knie, Sportfähigkeit wiedererlangt, Basistherapie mit Etanercept bis dato, 13-Jahreskontrolle ohne Lockerung, kein Synovialitisrezidiv ( Abb. 2).

\section{Fall 3}

51-jährige Frau mit seit dem fünften Lebensjahr bestehender HLA-B27-assoziierter Oligoarthritis. Verlauf als blande einge- stuft, alle gängigen Basistherapien unverträglich oder ineffektiv, im Alter von 41 Jahren Hüftgelenksersatz links, Dauerschmerzen beide unteren und oberen Sprunggelenke und rechter Ellenbogen sowie rechte Hüfte ( Abb. 3).

\section{Fall 4}

34-jährige JIA-Patientin seit dem 17. Lebensjahr im Rollstuhl, Entscheidung zum Ersatz von Knie- und Hüftgelenken, ausgeprägte Kontrakturen Hüfte und Knie beidseits, wegen Dysplasie beider Knie Individualplanung mit Sonderprothesen, Gehfähigkeit nach 4-Gelenksersatz und nach sechs Monaten erreicht. Standzeit der Endoprothesen bislang 15 Jahre ( Abb. 4). 

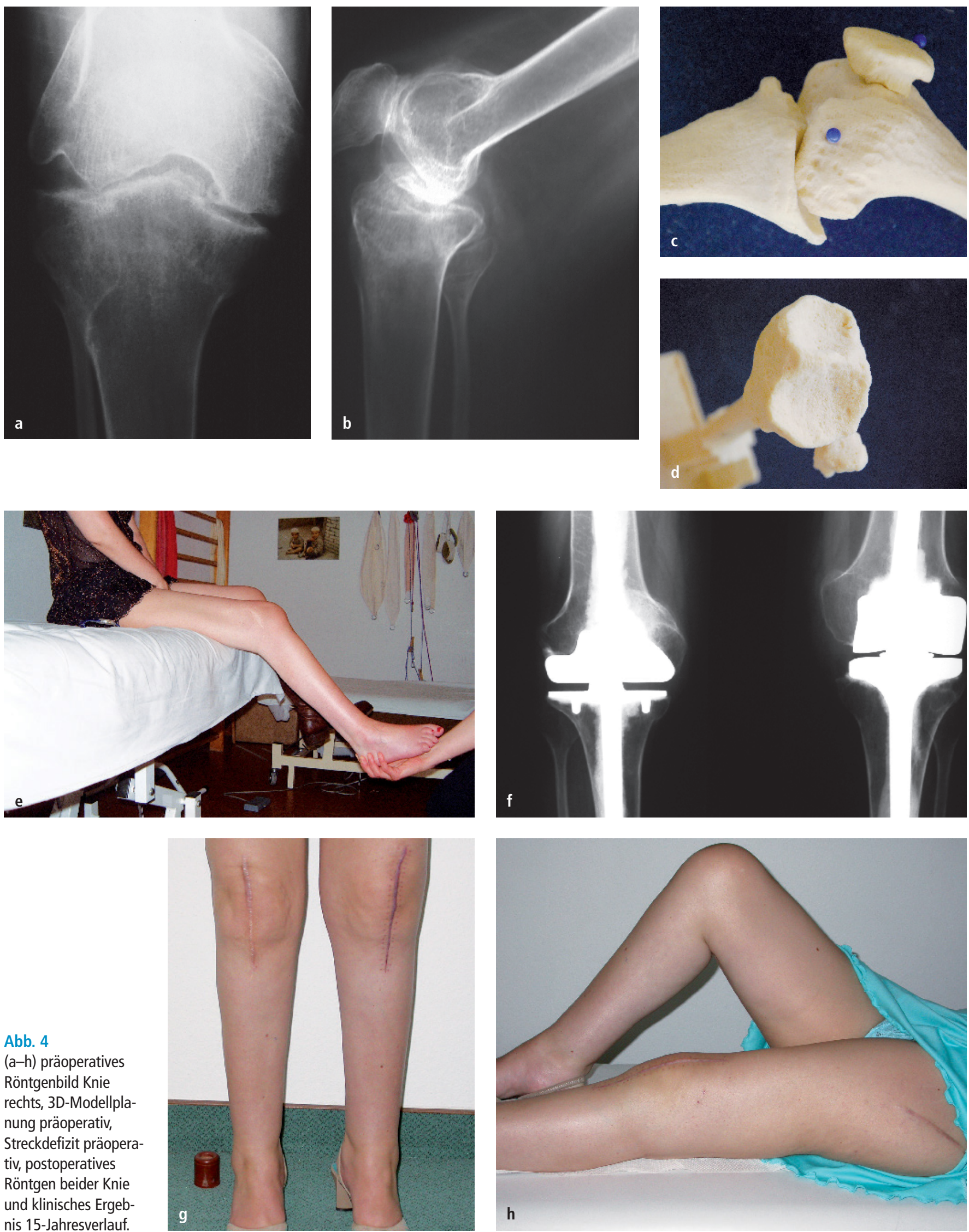

Abb. 4

(a-h) präoperatives Röntgenbild Knie rechts, 3D-Modellplanung präoperativ, Streckdefizit präoperativ, postoperatives Röntgen beider Knie und klinisches Ergebnis 15-Jahresverlauf.
9 

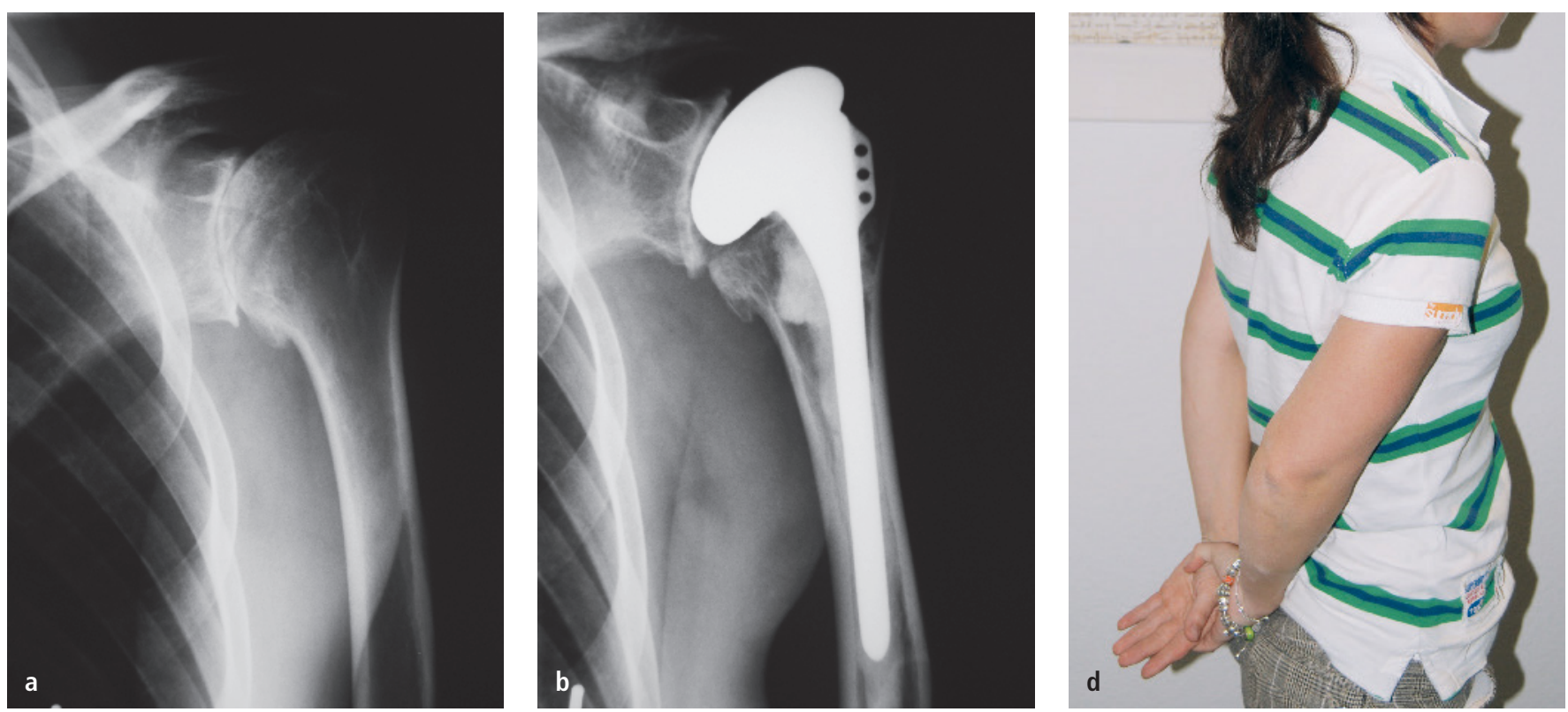

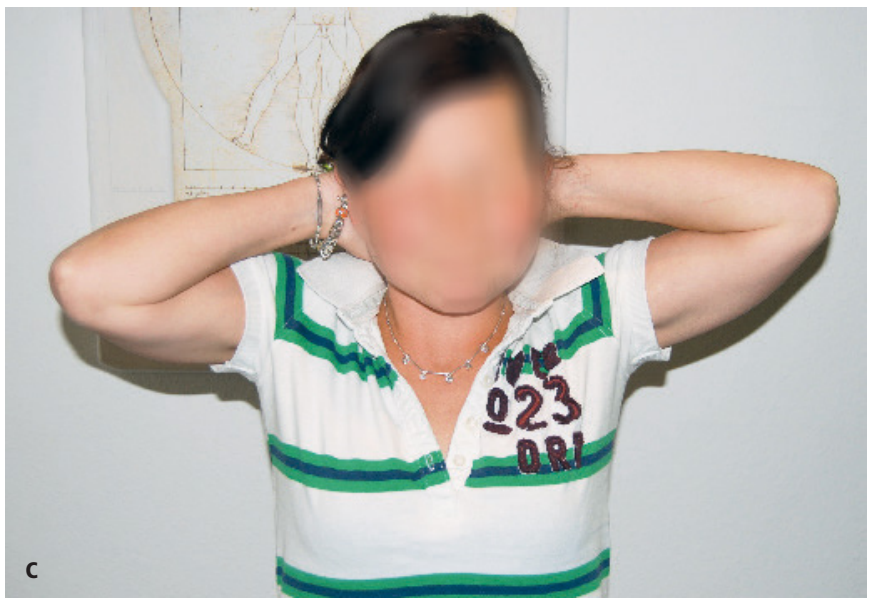

Abb. 5

(a-d) radiologisch linke Schulter präoperativ und postoperativ nach zwölf Jahren sowie klinisches Ergebnis

Fall 5

JIA seit dem sechsten Lebensjahr, polyartikulärer Verlauf unter Etanercept bei einer 34-jährigen Frau, sekundäre Destruktion beider Schultern mit hemiendoprothetischem Ersatz im Alter von 22 Jahren, zwölfjähriger Verlauf mit beginnender Dezentrierung radiologisch jedoch klinisch noch zufriedenstellendem Bewegungsumfang. Versteifung linkes unteres und rechtes oberes und unteres Sprunggelenk ( Abb. 5).

\section{Ergebnisse und Diskussion}

Der Schultergelenkbefall im Rahmen der JIA ist mit $50 \%(2,36)$ häufig, endoprothe- tische Versorgung doch eher gering. So beschreibt S. Thomas (36) eine 6-JahresNachbeobachtung nach Hemiarthroplastik der Schulter bei JIA. Bei im Mittel 32-Jährigen fand 27 Jahre nach Beginn der Erkrankung die operative Versorgung statt. Radiologische Lockerungen wurden bis dahin nicht beobachtet und Revisionen waren nicht erforderlich. Aufmerksam machen die Autoren auf die besonderen kontrakten Weichteilverhältnisse, Knochendeformitäten und die Notwendigkeit, eher kleine Implantatgrößen verwendet zu haben. Der "golden standard“ an der Schulter beim Rheumatiker ist die Hemiarthroplastik (2), wegen der nicht unerheblichen aseptischen Lockerungsraten von Pfannen bei den anatomischen Komplettendoprothesen im Vergleich(13). Ist die Rotatorenmanschette allerdings defekt oder besteht gar ein kompletter Cuffdefekt scheinen inverse Prothesen ein besseres Outcome zu haben. So muss bei den jungen Rheumatikern der Weichteilbefall primär mitbedacht werden, wenn die Planung zur endoprothetischen Versorgung ansteht. Revisionen der hemiarthroplastischen Stile mit Zement sind schwierig, weswegen die Planung primär eine metaphysär verankerte inverse Prothese zu implantieren durchaus für die Langzeitergebnisgestaltung gerechtfertigt erscheint (22).

Seltener, aber nicht weniger kompliziert, ist die Ellenbogenendoprothetik. So berichtet Baghdadi et al. (3) über 29 Ellenbogen in einem Beobachtungszeitraum von 22 Jahren mit einem Durchschnittsalter der Patienten von 37 Jahren. Wie bei den Schultern sind Schmerzbefreiung und Bewegungsgewinn eindrücklich, die KaplanMeier-Standzeiten bei der „semiconstrained" Version sind 96,4\% nach fünf Jahren und $79,9 \%$ nach zehn Jahren. In $17 \%$ wurden Individualimplantate verwandt (3).

Geringere Fallzahlen in langen Beobachtungszeiträumen erschweren die Interpretation der Ergebnisse, so steigt jedoch die Revisionsrate auch in anderen Studien bis zu 30-40\% nach über zehn Jahren, weswegen der Zeitpunkt der Implantation beim jüngeren noch sehr aktiven Rheumatiker mit äußerster Zurückhaltung getroffen werden sollte. 


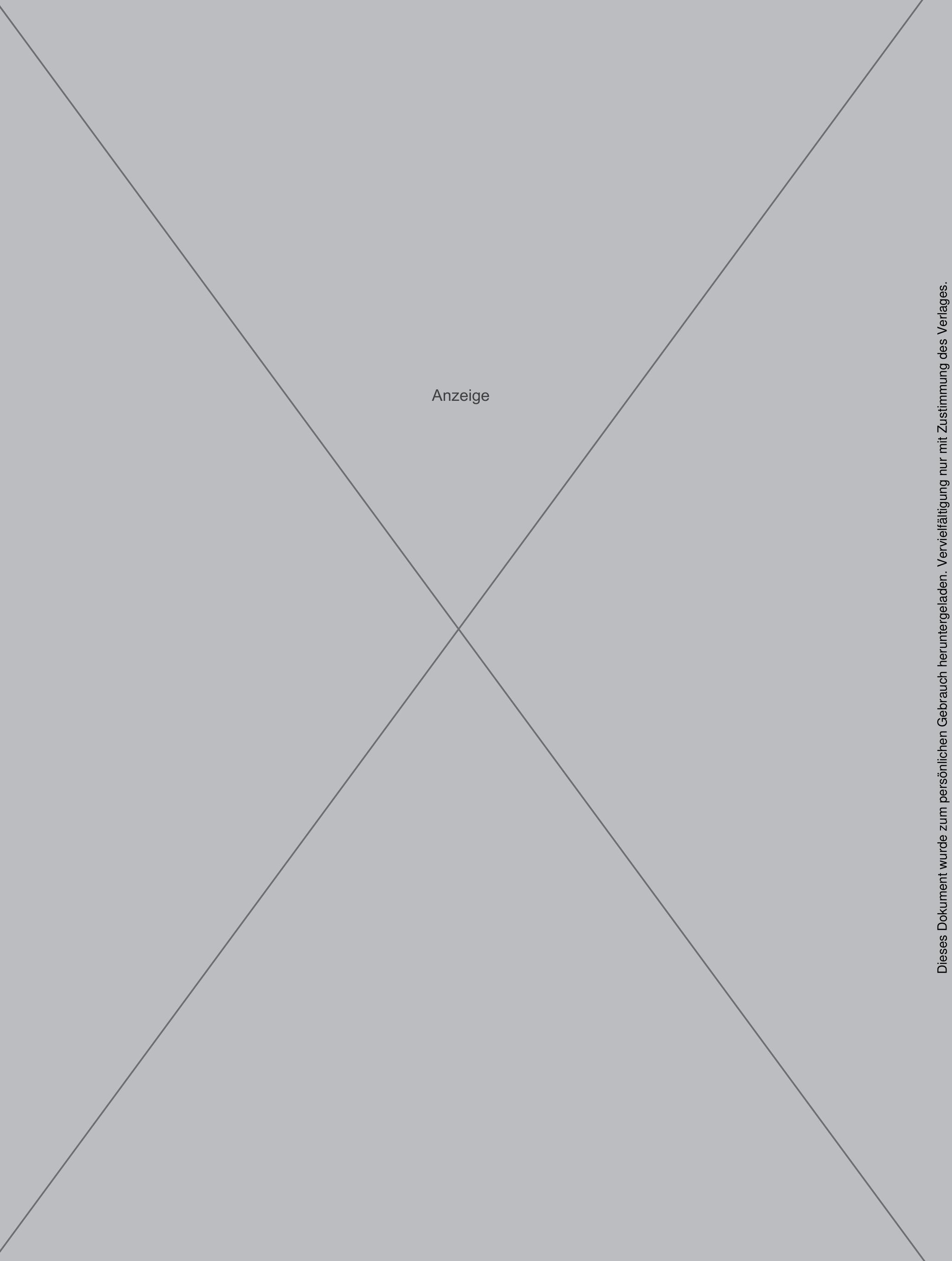




\section{Fazit}

Über die Hälfte der JIA-Patienten haben als Erwachsene weiterhin Probleme in den befallenen Gelenken. Aufgrund der verbesserten medikamentösen Therapiemöglichkeiten kann aber heutzutage die Gelenkdestruktion länger aufgehalten werden. Tendenziell sind es mehr polyartikuläre Verlaufsformen im Vergleich zu den oligoarthritischen oder monarthritischen Verläufen, die Therapiebedürftigkeit aufweisen. Mittelfristig können über die Endoprothetik zufriedenstellende Ergebnisse, insbesondere in der Knie- und Hüftendoprothetik, aber auch bereits in der Schulterendoprothetik erzielt werden. Insbesondere in der Schulterendoprothetik ist die Überlegung, perspektivisch eine metaphysäre Verankerung mit einer inversen Endoprothese einer Hemiprothese aufgrund der Weichteildegeneration vorzuziehen, ein wichtiger Aspekt. Eine Lebensplanung der jüngeren Klientel mit der Endoprothese erscheint heute insbesondere in Hüft- und Knieendoprothetik besser planbar als noch vor drei Jahrzehnten, allerdings verbunden mit komplexeren Revisionsoperationen. Diese ziehen an der Hüfte eine höhere Komplikationsrate nach sich als bei anderen Erkrankungen. Die Endoprothetik an Ellenbogen und Sprunggelenken ist Einzelfällen vorbehalten.

Die Handgelenksendoprothetik ist Einzelfällen vorbehalten und sollte die Möglichkeit auf einen Rückzug auf eine Handgelenksarthrodese immer miteinbeziehen.

An Fingergrund- und -mittelgelenken scheint die Silikon-Kautschuk-Alloarthroplastik von Swanson aus den 1960iger-Jahren immer noch „Goldstandard“ zu sein (35). Wechselraten zwischen sechs und 18 Jahren sind beschrieben (35).

Häufig wiederum ist der Hüftgelenksbefall, der üblicherweise mit Standardimplantaten versorgt werden kann. Techniken unterscheiden sich nicht wesentlich von den Vorgehensweisen bei der primären Koxarthrose (31). Die Ergebnisse sind im Hinblick auf Schmerzbefreiung, Funktionsverbesserung und Patientenzufriedenheit unabhängig von der Verankerungsart $(1,4)$ besser als präoperativ und zeigen nur gering schlechtere Ergebnisse als bei Arthro- sedaten (23). Standzeiten sind jedoch limitiert und zeigen Pfannenlockerungen im höheren Ausmaß als bei anderen Patienten (9). Auch ist im Revisionsfall die Komplikationsrate höher. So berichtet Goodman (9) über 24 Revisionen nach Hüftendoprothetik in 15 Fällen im Mittel nach neun Jahren bei einem Durchschnittsalter der Patienten von 35 (21-53) Jahren mit einer Komplikationsrate von 30\%. Allerdings berichten Adelani et al. von deutlich längeren Standzeiten nach 1988 im Vergleich zu den Jahren zuvor (1).

Gute Ergebnisse nach Knieendoprothetik zeigt Palmer 2005 auf. Fünfzehn Implantationen bei JIA zeigten nach im Minimum zwölf Jahren eine gute Schmerzrückbildung und funktionelle Verbesserung. Acht Patienten konnten dauerhaft auf den Rollstuhl verzichten und $40^{\circ}$ Bewegungsverbesserung waren $\mathrm{zu}$ verzeichnen (28). Überlebensraten von $97 \%$ nach sechs Jahren (6) oder 99\% nach fünf Jahren (23) bis 95\% nach zehn Jahren sind vergleichbar mit Ergebnissen aus nichtrheumatischen Fallstudien, allerdings 58,5\% nach 20 Jahren in einer Studie von Maviya et al. lassen die schlechtere Knochenqualität als einen wesentlichen Grund für die verminderte Standzeit heranziehen. Dennoch ist die dramatische Verbesserung der Lebensqualität in vielen Studien dokumentiert.

Die Spunggelenkendoprothetik bei der JIA ist wie die Prothetik des Handgelenkes Einzelfällen vorbehalten und sollte wegen der fehlenden Langzeitergebnisse mit Bedacht entschieden werden. Revisionen bis hin zur Arthrodese sind nicht ohne Komplikationen und bedeuten für die Betroffenen einen längeren Leidensweg.

\section{Interessenkonflikt}

Der korrespondierende Autor gibt an, dass kein Interessenkonflikt besteht.

\section{Literatur}

1. Adelani MA, Keeney JA, Palisch A et al. Has total hip arthroplasty in patients 30 years or younger improved? A systematic rewiew. Clin Orthop Relat Res 2013; 471 (8): 2595-2601.

2. Arbogast M. Surgery for juvenile idiapathic arthritis. In Benson, Fixen, Manicol, Parsch. $3^{\text {rd }}$ ed. Children's Orthopedics and Fractures. Springer 2010; 202-212.
3. Baghdadi YM, Jacobson JA, Duquin TR et al. The outcome of total elbow arthroplasty in juvenile idiopathic arthritis (juvenile rheumatoid arthritis) patients. J Shoulder Elbow Surg 2014; 23 (9): 1374-1380.

4. Bisel N, Gökce A, Kesmezacar H et al. Long -term results of total hip arthroplasty in patients with juvenile rheumatoid arthritis. Acta Orthop Traumatol Turc 2008; 42 (2): 119-245.

5. Bonnomet F, Glorion C. Total hip arthroplasty in patients younger than 30 years old: conclusions and recomendations. Rev Chir Orthop Reparatrice Appar Mot 2008; 94 (Suppl 6): 192-196.

6. Buchheit J, Serre A, Bouilloux X et al. Cementless total knee arthroplasty in chronic inflammatory rheumatism. Eur J Orthop Surg Traumatol 2014; 24 (8): 1489-1498.

7. Dophin B, Izem I, Dloin X et al. Total hip arthroplasty in patients younger than 30 years old with childhood rheumatic disease. Rev Chir Orthop Reparatrice Appar Mot 2008; 94 (Suppl 6): 149-153.

8. Dueckers G, Guellac N, Arbogast M et al. Evidence and consensus based treatment guidelines 2010 for juvenile idiopathic arthritis by the german society of paediatric rheumatology. Klin Padiatr 2011; 223: 386-394.

9. Goodman SB, Hwang K, Imrie S. High complication rate in revision total hip arthroplasty in juvenil idiopathic arthritis. Clin othop Relat Res 2014; 472 (2): 637-644.

10. Haas JP, Häfner R, Truckenbrodt H. Weitere rheumatische Erkankungen im Kindes- und Jugendalter. In: Manger B, Schulze-Koops H, Hrsg. Checkliste: Rheumatologie, 4. Aufl. Stuttgart New York: Georg Thieme 2012; 401-431.

11. Heyse TJ, Ries MD, Bellemans J et al. Total knee arthroplasty in patients with juvenile idiopathic arthritis. Clin Orthop Relat Res 2014; 472 (1): 147-154.

12. Hugle B, Haas JP, Benseler SM. Treatment preferences in juvenile idiopathic arthritis - a comparative analysis in two health care systems. Pediatric rheumatology online journal 2013; 11: 3.

13. Irlenbusch J, Irlenbusch U. Aktueller Stand der Schulterendoprothetik. Orthopädie und Unfallchirurgie up2date 2007; 4: 289-306.

14. Jolles BM, Grosso P, Bogoch ER. Shoulder arthroplasty for patients with juvenile idiopathic arthritis. J arthroplasty 2007; 22 (6): 876-883.

15. Jolles BM, Bogoch ER. Quality of life after TKA für patients with juvenile idiopathic arthritis. Clin Orthop Relat Res 2008; 466 (1): 167-178.

16. Kim HJ, Kahn B, Figgie MP. Total joint replacement in childhood arthritis. Curr rheumatol Rep 2008; 10 (2): 135-141.

17. Kitsoulis PB, Siamopoulou A, Beris AE, Xenakis TA. Total hip and knee arthroplasty for juvenile rheumatoid arthritis. Folia Med (Plovdiv) 2006; 48 (3-4): 42-49.

18. Kitsoulis PB, Stafilas KS, Siamopoulou A et al. Total hip arthroplasty in children with juvenile chronic arthritis: long-term results. J Pediatr Orthop 2006; 26 (1): 8-12.

19. Krüger K, Albrecht K, Rehart S, Scholz R. Empfehlungen der DGRh zur perioperativen Vorgehensweise unter Therapie mit DMARD und Biologicals bei Entzündlich-rheumatischen Erkrankungen. Z Rheumatol 2014. 
20. Krumrey-Langkammerer M, Häfner R. Evaluation of the ILAR criteria for juvenile idiopathic arthritis. The Journal of rheumatology 2001; 28: 2544-2547.

21. Larsen A, Dale K, Eek M .Radiographic evaluation of rheumatoid arthritis and related conditions by standard reference films. Acta Radiol Diagnosis 1977; 18: 481-491.

22. Levy O, Narvani A, Hous $\mathrm{N}$ et al. Reverse shoulder arthroplasty with a cementless short metaphyseal humeral implant without a stem: clinical and radiologic outcomes in prospective 2- to 7-year followup study. J Shoulder Elbow Surg 2016; 25 (8): 1362-1370

23. Li XA, Iyer S, Cross MB, Figgie MP. Total joint replacement in adolescents: literature review and case examples. Curr Opin Pediatr 2012; 24 (1): $57-63$.

24. Lybäck CO, Belt EA, Hämäläinen MM et al. Survivorship of AGC knee replacement in juvenile chronic arthritis: 13-year follow-up of 77 knees. J Arthroplasty 2000; 15 (2): 166-170.

25. Malviya A, Forster HE, Avery P et al. Long term outcome following knee replacement in patients with juvenile idiopatic arthritis. Knee 2010; 17 (5): 340-344.
26. Mertelsmann-Voss C, Lyman S, Pan TJ et al. US trends in rates of arthroplasty for inflammatory arthritis including rheumatoid arthritis, juvenile idiopathic arthritis and spondyloarthritis. Arthritis Rheumatol 2014; 66 (6): 1432-1439.

27. Michels H, Ganser G, Dannecker G et al. Structural quality of rheumatology clinics for children and adolescents. Paper by a task force of the „Society of Pediatric and Adolescent Rheumatology" and of the "Association of Rheumatology Clinics in Germany “. Zeitschrift fur Rheumatologie 2006; 65: 315-322, 324-316.

28. Palmer DH, Mulhall KJ, Thompson CA et al. Total knee arthroplasty in juvenile rheumatoid arthritis. J Bone Joint Surg Am 2005; 87 (7): 1510-1514.

29. Parvizi J, Lajam CM, Trousdale RT et al. Total knee arthroplasty in young patients with juvenile rheumatoid arthritis. J Bone Joint Srg Am 2003; 85-A (6): 1090-1094

30. Petty RE, Southwood TR, Baum J et al. Revision of the proposed classification criteria for juvenile idiopathic arthritis: Durban, 1997. The Journal of rheumatology 1998; 25: 1991-1994.

31. Rehart S, Henninger M. Die endoprothetische Versorgung zerstörter Hüftgelenke bei juvenilen rheumatischen Erkrankungen. OUP 2016; 6: 364-369.
32. Restrepo C, Lettich T, Roberts N et al. Uncemented total hip arthroplasty in patients less than twentyyears. Acta orthop Belg 2008; 74 (5): 615-622.

33. Rojer DE, Goodman SB. Total knee replacement in juvenile rheumatoid arthritis. Orthopedics 2005; 28 (1): 39-45.

34. Schenk K, Lieske S, John M et al. Prospective study of a cementless, mobile-bearing third generation total ankle prosthesis. Foot ankle Int 2011; 32 (8): 755-763.

35. Schmidt K. Versorgung der Fingergelenke. Akt Rheumatol 2014; 39: 106-113.

36. Thomas S, Price AJ, Sankey RA, Thomas M. Shoulder hemiarthroplasty in patients with juvenile idiaopathic arthritis. J Bone Joint Surg Br 2005; 87 (5): 672-676.

37. Thomas A, Rojer D, Imrie S, Goodman SB. Cemented total knee arthroplasty in patients with juvenile rheumatoid arthitis. Clin orthop Relat Res 2005; 433: 140-146.

38. Wanivenhaus A. Handgelenksprothese. Akt rheumatol 2014; 39: 100-105.

39. Zwartelé RE, Witjes S, Doets HC et al. Cementless total hip arthroplasty in rheumatoid arthritis: a systematic review of the literature. Arch Orthop Trauma Surg 2012; 132 (4): 535-546. Epub 2011 Nov 24. 